\title{
LEARNING ENGINEERING DESIGN THROUGH MULTIMEDIA CASE STUDIES
}

\author{
D. Effa*; S. Lambert**: O. Nespoli*** \\ Waterloo Cases in Design Engineering, University of Waterloo, Ontario Canada \\ deffa@uwaterloo.ca*, steve@uwaterloo.ca**,oscar@uwaterloo.ca***
}

\section{INTRODUCTION}

Providing appropriate context is a vital element for teaching and learning. An excellent way of achieving this is through case studies: a description of actual engineering projects carried out in industry. Engineering design case studies could provide students examples of many practical and accessible real-life principles which are representative of situations engineers face in their professional life. The Waterloo Cases in Design Engineering (WCDE) group at the University of Waterloo has been established to promote case methods by generating engineering design case studies, and implementing them throughout the engineering faculty [1].

While the majority of WCDE cases are text-based, cases with multimedia content have been shown to be an effective way of documenting and teaching best design practices. Multimedia cases can add depth and interest to classroom discussions, and better address different student learning styles [2]. Recently, WCDE has placed more emphasis on the power of multimedia, to take advantage of the unique message-carrying ability of video, simulation, and graphics, to enhance the already powerful case method. This paper highlights the current process for updating and enhancing the current implementation of the case method.

\section{WCDE MULTIMEDIA CASES}

The application of multimedia teaching materials has been advocated increasingly in the educational technology literature [3]. Multimedia case studies illustrating engineering industrial practice can play a major role in boosting students' retention rate, stimulating their creativity, and broadening their understanding. WCDE has created and integrated multimedia tools into case studies for a number of courses. Currently available multimedia tools include case study background information, footage of industrial examples, experimental results, and computer simulations, Figure 1. In addition, students are exposed to physical hardware and computational software applications.

Time-lapse video of a cabinet maker assembling kitchen cabinets is an effective way of providing an opportunity for students to more fully understand and empathize with a situation, and to identify the real needs and most significant opportunities for improvement. Multimedia content is much in demand by students. In the recent implementation of a case study on the design of the battery pack for a hybrid electric vehicle, students were excited by the real-world application, but requested video to illustrate the actual hardware and applications.

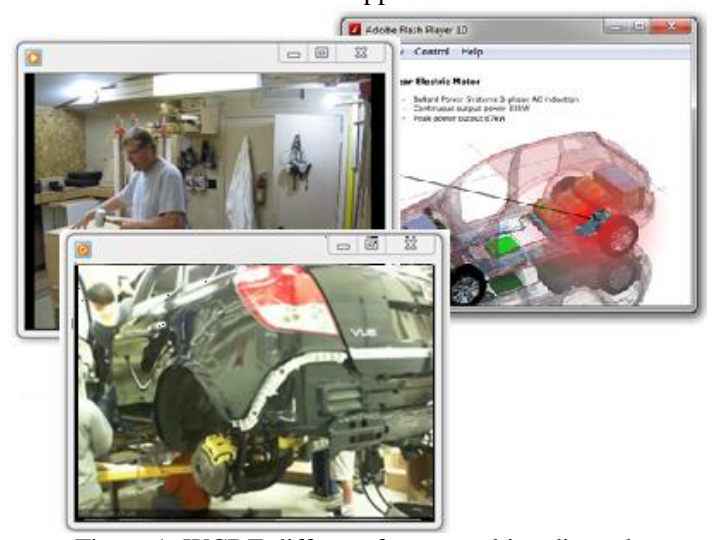

Figure 1: WCDE different format multimedia tool

\section{CURRENT STATUS AND FUTURE DEVELOPMENTS}

By illustrating the relevance and importance of all types of information, multimedia can add depth and interest to classroom lectures. WCDE at present is developing an Engineering Case Multimedia System (ECMS) to integrate the different teaching tools used in class for online and tablet PC applications. As Tablet PC's become more powerful and prevalent, they are expected to play an increasing role in the teaching and learning process. To take full advantage of these computational tools requires a new approach. The main goal of the ECMS project is to create a platform that is accessible to academics and industry professionals and that provides an easy to use and learn multimedia interface, as shown in Figure 2. Another important aspect of this platform is to assists online case implementation by minimizing instructor time, which is expected to be a major roadblock to changing the paradigm from classical lectures to add the case method approach into the class room.

One way of achieving this is to develop a catalog of multimedia (flash) presentations of cases along with other teaching materials that highlight different aspects of engineering practice. The use of multimedia flash presentations of case studies is an excellent means of illustrating engineering science theory in conjunction with real examples from industry. The advantage of capturing the case studies in this format is that once they have been developed, they can be used repeatedly and in different contexts. Flash presentation has been selected mainly because it has the capacity to integrate a wide range of multimedia formats into one complete package. Such a platform would do the following:

- Allow case authors to leverage the digital medium by supporting multimedia formats such as images, audio, video, animation, and simulation

- Assist online case implementation

- Package case content into a visually appealing and well-organized case study

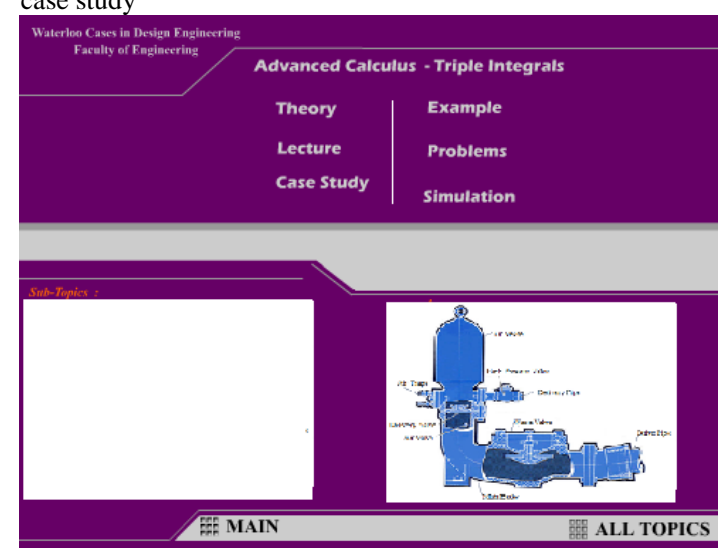

Figure 2: Multimedia flash presentations platform

\section{CONCLUSION}

Making linkages between engineering theory and practice is important for student motivation and learning. The use of multimedia case studies is one approach for achieving this, as it enables students to see the relevance of what they are learning in a real engineering context. The proposed interactive multimedia formats engage students in active thought while exploring the design case study. Video, sound, animation, graphics, and text are integrated to provide an ideal medium for exploring engineering disciplines. In these cases, students can review how exemplary products are conceived, how products are designed for easy manufacturing and assembly, as well as study issues in reliability and maintenance.

\section{REFERENCES}

1. O. Nespoli and S. Lambert, "Engineering Case Studies: Effective and Sustainable Development Methods", ASEE 2010 Annual Conference, Louisville Kentucky, June 20-23.

2. R.M. Felder, R. Brent, and M.J. Prince, "Engineering Instructional Development: Programs, Best Practices, an Recommendations", Journal of Engineering Education, Vol. 100, No. 1, pp890122.

3. Ashok Kumar Manoharan, P.K. Raju \& Chetan Sankar, " Use of Multimedia Case Studies in an Introductory Course in Mechanical Engineering", ASEE 2010 